\title{
Discrete-time bifurcation behavior of a prey-predator system with generalized predator
}

\author{
Harkaran Singh ${ }^{1 *}$, Joydip Dhar ${ }^{2}$ and Harbax Singh Bhatti ${ }^{3}$
}

\section{"Correspondence:}

harkaran78@yahoo.in

'Department of Applied Sciences,

Khalsa College of Engineering and

Technology, Amritsar, Punjab

143001, India

Full list of author information is

available at the end of the article

\begin{abstract}
In the present study, keeping in view of Leslie-Gower prey-predator model, the stability and bifurcation analysis of discrete-time prey-predator system with generalized predator (i.e., predator partially dependent on prey) is examined. Global stability of the system at the fixed points has been discussed. The specific conditions for existence of flip bifurcation and Neimark-Sacker bifurcation in the interior of $R_{+}^{2}$ have been derived by using center manifold theorem and bifurcation theory. Numerical simulation results show consistency with theoretical analysis. In the case of a flip bifurcation, numerical simulations display orbits of period 2, 4, 8 and chaotic sets; whereas in the case of a Neimark-Sacker bifurcation, a smooth invariant circle bifurcates from the fixed point and stable period 16, 26 windows appear within the chaotic area. The complexity of the dynamical behavior is confirmed by a computation of the Lyapunov exponents.
\end{abstract}

Keywords: prey-predator system; center manifold theorem; flip bifurcation; Neimark-Sacker bifurcation; Lyapunov exponent; chaos

\section{Introduction}

It is a well recognized fact that the prey-predator interaction is a subject of great interest in the bio-mathematical literature and the dynamic relationship between predator and prey living in the same environment will continue to be one of the important themes in mathematical ecology (Berryman [1], Lotka [2], May [3], Volterra [4]). Many researchers studied the dynamical behavior of the prey-predator system in ecology and contributed to the growth of the population models [2-24].

Liu [25] investigated the existence of periodic solutions for a discrete semi-ratiodependent prey-predator model. Huo and Li [26] obtained conditions for the global stability of solutions for a delayed discrete prey-predator system with the help of Lyapunov functions. Chen [27] proposed a discrete prey-predator system and obtained conditions for the global stability of an equilibrium for non-autonomous and periodic cases. Liao et al. [28] investigated a one-predator two-prey discrete model and derived the conditions for the local asymptotic stability of equilibrium of the system. Fan and Li [29] established sufficient conditions in a delayed discrete prey-predator model with Holling type III functional response for permanence. However, there are few articles discussing the dynamical

(c) 2015 Singh et al. This article is distributed under the terms of the Creative Commons Attribution 4.0 International License (http://creativecommons.org/licenses/by/4.0/), which permits unrestricted use, distribution, and reproduction in any medium, provided you give appropriate credit to the original author(s) and the source, provide a link to the Creative Commons license, and indicate if changes were made. 
behavior of discrete-time prey-predator models for exploring the possibility of bifurcations and chaos phenomena [30-35].

In the present study, motivated by the Leslie-Gower prey-predator model [36, 37], we propose a discrete-time prey-predator system with predator partially dependent on prey [38] and investigate the stability and bifurcation analysis of the system by using center manifold theorem and bifurcation theory. This paper is organized as follows: in Section 2, we obtained fixed points of the discrete-time system and discussed the stability criterion of the system at the fixed points. In Section 3, the specific conditions of the existence of a flip bifurcation and a Neimark-Sacker bifurcation are derived. Finally, in Section 4, numerical simulations are carried out to support our analytical findings, especially for period doubling bifurcation and chaotic behavior.

The prey-predator system is of the form

$$
\left\{\begin{array}{l}
\frac{d x}{d t}=a x\left(1-\frac{x}{k}\right)-\frac{b x y}{x+l} \\
\frac{d y}{d t}=\left[1-\frac{m y}{n x+q}-d\right] y
\end{array}\right.
$$

where $x(t)$ and $y(t)$ represent the densities of prey and predator populations, respectively. Again, the parameter $a$ denote the intrinsic growth rate of prey; $b$ is harvesting rate of prey by predator; $d$ denotes the death rate of predator; $k$ denotes carrying capacity of the prey in a particular habitat; $l$ denotes the half saturation constant; $m$ is the maximum value which per capita reduction rate of predator can attend; $n$ is a measure of the food quality that the prey provides for conversion into predator births; $q$ is the extent to which alternatives are provided for the growth of predator.

Applying the forward Euler scheme to the system of equations (1), we obtain the discrete-time prey-predator system:

$$
\left\{\begin{array}{l}
x \rightarrow x+\delta\left[a x\left(1-\frac{x}{k}\right)-\frac{b x y}{x+l}\right] \\
y \rightarrow y+\delta\left[1-\frac{m y}{n x+q}-d\right] y
\end{array}\right.
$$

where $\delta$ is the step size. The numerical solution to the initial value problem obtained from Euler's method with step size $\delta$, and the total number of steps $N_{0}$ satisfies $0<\delta \leq \frac{L_{0}}{N_{0}}$, where $L_{0}$ is the length of the interval.

\section{Stability of fixed points}

The fixed points of the system (2) are $O(k, 0), A\left(0, \frac{(1-d) q}{m}\right)$ and $B\left(x^{*}, y^{*}\right)$, where $x^{*}, y^{*}$ satisfy

$$
\left\{\begin{array}{l}
a\left(1-\frac{x^{*}}{k}\right)-\frac{b y^{*}}{x^{*}+l}=0, \\
1-\frac{m y^{*}}{n x^{*}+q}-d=0 .
\end{array}\right.
$$

The Jacobian matrix of (2) at the fixed point $(x, y)$ is written as

$$
J=\left[\begin{array}{cc}
1+\delta\left(a-\frac{2 a x}{k}-\frac{b l y}{(x+l)^{2}}\right) & -\frac{b \delta x}{(x+l)} \\
\frac{\delta m n y^{2}}{(n x+q)^{2}} & 1+\delta\left(1-\frac{2 m y}{n x+q}-d\right)
\end{array}\right] .
$$

The characteristic equation of the Jacobian matrix is given by

$$
\lambda^{2}+p(x, y) \lambda+q(x, y)=0
$$


where

$$
\begin{aligned}
p(x, y)= & -\operatorname{tr} J=-2-\delta\left(1+a-d-\frac{2 a x}{k}-\frac{b l y}{(x+l)^{2}}-\frac{2 m y}{n x+q}\right), \\
q(x, y)= & \operatorname{det} J \\
= & {\left[1+\delta\left(a-\frac{2 a x}{k}-\frac{b l y}{(x+l)^{2}}\right)\right]\left[1+\delta\left(1-\frac{2 m y}{n x+q}-d\right)\right] } \\
& +\frac{\delta^{2} b m n x y^{2}}{(x+l)(n x+q)^{2}} .
\end{aligned}
$$

Now, we state a lemma similar to $[24,32]$.

Lemma 2.1 Let $F(\lambda)=\lambda^{2}+B \lambda+C$. Suppose that $F(1)>0 ; \lambda_{1}$ and $\lambda_{2}$ are roots of $F(\lambda)=0$. Then we have:

(i) $\left|\lambda_{1}\right|<1$ and $\left|\lambda_{2}\right|<1$ if and only if $F(-1)>0$ and $C<1$;

(ii) $\left|\lambda_{1}\right|<1$ and $\left|\lambda_{2}\right|>1$ (or $\left|\lambda_{1}\right|>1$ and $\left.\left|\lambda_{2}\right|<1\right)$ if and only if $F(-1)<0$;

(iii) $\left|\lambda_{1}\right|>1$ and $\left|\lambda_{2}\right|>1$ if and only if $F(-1)>0$ and $C>1$;

(iv) $\lambda_{1}=-1$ and $\left|\lambda_{2}\right| \neq 1$ if and only if $F(-1)=0$ and $B \neq 0,2$;

(v) $\lambda_{1}$ and $\lambda_{2}$ are complex and $\left|\lambda_{1}\right|=\left|\lambda_{2}\right|=1$ if and only if $B^{2}-4 C<0$ and $C=1$.

Let $\lambda_{1}$ and $\lambda_{2}$ be the roots of (4), which are known as eigen values of the fixed point $(x, y)$. The fixed point $(x, y)$ is a sink or locally asymptotically stable if $\left|\lambda_{1}\right|<1$ and $\left|\lambda_{2}\right|<1$. The fixed point $(x, y)$ is a source or locally unstable if $\left|\lambda_{1}\right|>1$ and $\left|\lambda_{2}\right|>1$. The fixed point $(x, y)$ is non-hyperbolic if either $\left|\lambda_{1}\right|=1$ or $\left|\lambda_{2}\right|=1$. The fixed point $(x, y)$ is a saddle if $\left|\lambda_{1}\right|>1$ and $\left|\lambda_{2}\right|<1$ (or $\left|\lambda_{1}\right|<1$ and $\left|\lambda_{2}\right|>1$ ).

Proposition 2.2 The fixed point $O(k, 0)$ is source if $\delta>\frac{2}{a}$, saddle if $0<\delta<\frac{2}{a}$, and nonhyperbolic if $\delta=\frac{2}{a}$.

One can see that when $\delta=\frac{2}{a}$, one of the eigen values of the fixed point $O(k, 0)$ is -1 and magnitude of other is not equal to 1 . Thus the flip bifurcation occurs when the parameter changes in a small neighborhood of $\delta=\frac{2}{a}$.

Proposition 2.3 There exist different topological types of $A\left(0, \frac{(1-d) q}{m}\right)$ for possible parameters.

(i) $A\left(0, \frac{(1-d) q}{m}\right)$ is $\operatorname{sink}$ if $b q(1-d)>$ alm and $0<\delta<\min \left\{\frac{2}{1-d}, \frac{2 l m}{b q(1-d)-a l m}\right\}$.

(ii) $A\left(0, \frac{(1-d) q}{m}\right)$ is source if $b q(1-d)>$ alm and $\delta>\max \left\{\frac{2}{1-d}, \frac{2 l m}{b q(1-d)-a l m}\right\}$.

(iii) $A\left(0, \frac{(1-d) q}{m}\right)$ is non-hyperbolic if $b q(1-d)>$ alm and either $\delta=\frac{2}{1-d}$ or $\delta=\frac{2 l m}{b q(1-d)-a l m}$.

(iv) $A\left(0, \frac{(1-d) q}{m}\right)$ is saddle for all values of the parameters, except for that values which lies in (i) to (iii).

The term (iii) of Proposition 2.3 implies that the parameters lie in the set

$$
\begin{aligned}
F_{A}= & \left\{(a, b, d, k, l, m, n, q, \delta), \delta=\frac{2}{1-d}, \delta \neq \frac{2 l m}{b q(1-d)-a l m}\right. \text { and } \\
& b q(1-d)>a l m, a, b, d, k, l, m, n, q, \delta>0\} .
\end{aligned}
$$


If the term (iii) of Proposition 2.3 holds, then one of the eigen values of the fixed point $A\left(0, \frac{(1-d) q}{m}\right)$ is -1 and the magnitude of the other is not equal to 1 . The point $A\left(0, \frac{(1-d) q}{m}\right)$ undergoes a flip bifurcation when the parameter changes in small neighborhood of $F_{A}$.

The characteristic equation of the Jacobian matrix $J$ of the system (2) at the fixed point $B\left(x^{*}, y^{*}\right)$ is written as

$$
\lambda^{2}+p\left(x^{*}, y^{*}\right) \lambda+q\left(x^{*}, y^{*}\right)=0,
$$

where

$$
\begin{aligned}
& p\left(x^{*}, y^{*}\right)=-2-G \delta, \\
& q\left(x^{*}, y^{*}\right)=1+G \delta+H \delta^{2}
\end{aligned}
$$

and

$$
\begin{aligned}
& G=1+a-d-\frac{2 a x^{*}}{k}-\frac{b l y^{*}}{\left(x^{*}+l\right)^{2}}-\frac{2 m y^{*}}{n x^{*}+q}, \\
& H=\left[a-\frac{2 a x^{*}}{k}-\frac{b l y^{*}}{\left(x^{*}+l\right)^{2}}\right]\left[1-\frac{2 m y^{*}}{n x^{*}+q}-d\right]+\frac{b m n x^{*} y^{* 2}}{\left(x^{*}+l\right)\left(n x^{*}+q\right)^{2}} .
\end{aligned}
$$

Now

$$
F(\lambda)=\lambda^{2}-(2+G \delta) \lambda+\left(1+G \delta+H \delta^{2}\right) .
$$

Therefore

$$
F(1)=H \delta^{2}, \quad F(-1)=4+2 G \delta+H \delta^{2} .
$$

Using Lemma 2.1, we get the following proposition.

Proposition 2.4 There exist different topological types of $B\left(x^{*}, y^{*}\right)$ for all possible parameters.

(i) $B\left(x^{*}, y^{*}\right)$ is a sink if either condition (i.1) or (i.2) holds:

(i.1) $G^{2}-4 H \geq 0$ and $0<\delta<\frac{-G-\sqrt{G^{2}-4 H}}{H}$,

(i.2) $G^{2}-4 H<0$ and $0<\delta<-\frac{G}{H}$.

(ii) $B\left(x^{*}, y^{*}\right)$ is source if either condition (ii.1) or (ii.2) holds:

(ii.1) $G^{2}-4 H \geq 0$ and $\delta>\frac{-G+\sqrt{G^{2}-4 H}}{H}$,

(ii.2) $G^{2}-4 H<0$ and $\delta>-\frac{G}{H}$.

(iii) $B\left(x^{*}, y^{*}\right)$ is non-hyperbolic if either condition (iii.1) or (iii.2) holds:

(iii.1) $G^{2}-4 H \geq 0$ and $\delta=\frac{-G \pm \sqrt{G^{2}-4 H}}{H}$,

(iii.2) $G^{2}-4 H<0$ and $\delta=-\frac{G}{H}$.

(iv) $B\left(x^{*}, y^{*}\right)$ is saddle for all values of the parameters, except for that values which lies in (i) to (iii).

If the term (iii.1) of Proposition 2.4 holds, then one of the eigen values of the fixed point $B\left(x^{*}, y^{*}\right)$ is -1 and the magnitude of the other is not equal to 1 . The term (iii.1) of Proposi- 
tion 2.4 may be written as follows:

$$
\begin{aligned}
F_{B 1}= & \left\{(a, b, d, k, l, m, n, q, \delta): \delta=\frac{-G-\sqrt{G^{2}-4 H}}{H}, G^{2}-4 H \geq 0\right. \text { and } \\
& a, b, d, k, l, m, n, q, \delta>0\} . \\
F_{B 2}= & \left\{(a, b, d, k, l, m, n, q, \delta): \delta=\frac{-G+\sqrt{G^{2}-4 H}}{H}, G^{2}-4 H \geq 0\right. \text { and } \\
& a, b, d, k, l, m, n, q, \delta>0\} .
\end{aligned}
$$

If the term (iii.2) of Proposition 2.4 holds, then the eigen values of the fixed point $B\left(x^{*}, y^{*}\right)$ are a pair of complex conjugate numbers with modulus 1 . The term (iii.2) of Proposition 2.4 may be written as follows:

$$
H_{B}=\left\{(a, b, d, k, l, m, n, q, \delta): \delta=-\frac{G}{H}, G^{2}-4 H<0 \text { and } a, b, d, k, l, m, n, q, \delta>0\right\} .
$$

\section{Bifurcation behavior}

In this section, we study the flip bifurcation and the Neimark-Sacker bifurcation at the fixed point $B\left(x^{*}, y^{*}\right)$.

\subsection{Flip bifurcation}

Consider the system (2) with arbitrary parameter $\left(a, b, d, k, l, m, n, q, \delta_{1}\right) \in F_{B 1}$, which is described as follows:

$$
\left\{\begin{array}{l}
x \rightarrow x+\delta_{1}\left[a x\left(1-\frac{x}{k}\right)-\frac{b x y}{x+l}\right] \\
y \rightarrow y+\delta_{1}\left[1-\frac{m y}{n x+q}-d\right] y .
\end{array}\right.
$$

$B\left(x^{*}, y^{*}\right)$ is fixed point of the system (6), where $x^{*}, y^{*}$ are given by (3) and

$$
\delta_{1}=\frac{-G-\sqrt{G^{2}-4 H}}{H} .
$$

The eigen values of $B\left(x^{*}, y^{*}\right)$ are $\lambda_{1}=-1, \lambda_{2}=3+G \delta_{1}$ with $\left|\lambda_{2}\right| \neq 1$ by Proposition 2.4. Consider the perturbation of (6)

$$
\left\{\begin{array}{l}
x \rightarrow x+\left(\delta_{1}+\delta^{*}\right)\left[a x\left(1-\frac{x}{k}\right)-\frac{b x y}{x+l}\right] \\
y \rightarrow y+\left(\delta_{1}+\delta^{*}\right)\left[1-\frac{m y}{n x+q}-d\right] y
\end{array}\right.
$$

where $\left|\delta^{*}\right| \ll 1$ is a limited perturbation parameter.

Let $u=x-x^{*}$ and $v=y-y^{*}$.

After the transformation of the fixed point $B\left(x^{*}, y^{*}\right)$ of the system (7) to the point $(0,0)$, we obtain

$$
\left(\begin{array}{c}
u \\
v
\end{array}\right) \rightarrow\left(\begin{array}{c}
a_{11} u+a_{12} v+a_{13} u^{2}+a_{14} u v+b_{11} \delta^{*} u+b_{12} \delta^{*} v \\
+b_{13} \delta^{*} u^{2}+b_{14} \delta^{*} u v+O\left(|u|,|v|,\left|\delta^{*}\right|\right)^{3} \\
a_{21} u+a_{22} v+a_{23} u^{2}+a_{24} u v+a_{25} v^{2}+b_{21} \delta^{*} u+b_{22} \delta^{*} v \\
+b_{23} \delta^{*} u^{2}+b_{24} \delta^{*} u v+b_{25} \delta^{*} v^{2}+O\left(|u|,|v|,\left|\delta^{*}\right|\right)^{3}
\end{array}\right)
$$


where

$$
\begin{aligned}
& a_{11}=1+\delta_{1}\left[-\frac{a}{k} x^{*}+\frac{b x^{*} y^{*}}{\left(x^{*}+l\right)^{2}}\right], \quad a_{12}=-\frac{b \delta_{1} x^{*}}{x^{*}+l}, \\
& a_{13}=\delta_{1}\left[-\frac{a}{k}+\frac{b l y^{*}}{\left(x^{*}+l\right)^{3}}\right], \quad a_{14}=-\frac{\delta_{1} b l}{\left(x^{*}+l\right)^{2}}, \\
& b_{11}=-\frac{a}{k} x^{*}+\frac{b x^{*} y^{*}}{\left(x^{*}+l\right)^{2}}, \quad b_{12}=-\frac{b x^{*}}{x^{*}+l}, \\
& b_{13}=-\frac{a}{k}+\frac{b l y^{*}}{\left(x^{*}+l\right)^{3}}, \quad b_{14}=-\frac{b l}{\left(x^{*}+l\right)^{2}}, \\
& a_{21}=\frac{\delta_{1} m n y^{* 2}}{\left(n x^{*}+q\right)^{2}}, \quad a_{22}=1+\delta_{1}(1-d)-\frac{2 \delta_{1} m y^{*}}{n x^{*}+q}, \\
& a_{23}=-\frac{\delta_{1} m n^{2} y^{* 2}}{\left(n x^{*}+q\right)^{3}}, \quad a_{24}=\frac{2 \delta_{1} m n y^{*}}{\left(n x^{*}+q\right)^{2}}, \quad a_{25}=-\frac{\delta_{1} m}{n x^{*}+q}, \\
& b_{21}=\frac{m n y^{* 2}}{\left(n x^{*}+q\right)^{2}}, \quad b_{22}=1-d-\frac{2 m y^{*}}{n x^{*}+q}, \quad b_{23}=-\frac{m n^{2} y^{* 2}}{\left(n x^{*}+q\right)^{3}}, \\
& b_{24}=\frac{2 m n y^{*}}{\left(n x^{*}+q\right)^{2}}, \quad b_{25}=-\frac{m}{n x^{*}+q} .
\end{aligned}
$$

Consider the following translation:

$$
\left(\begin{array}{l}
u \\
v
\end{array}\right)=T\left(\begin{array}{l}
\tilde{x} \\
\tilde{y}
\end{array}\right),
$$

where

$$
T=\left(\begin{array}{cc}
a_{12} & a_{12} \\
-1-a_{11} & \lambda_{2}-a_{11}
\end{array}\right) .
$$

Taking $T^{-1}$ on both sides of (8), we get

$$
\left(\begin{array}{l}
\tilde{x} \\
\tilde{y}
\end{array}\right) \rightarrow\left(\begin{array}{cc}
-1 & 0 \\
0 & \lambda_{2}
\end{array}\right)\left(\begin{array}{l}
\tilde{x} \\
\tilde{y}
\end{array}\right)+\left(\begin{array}{l}
f\left(u, v, \delta^{*}\right) \\
g\left(u, v, \delta^{*}\right)
\end{array}\right),
$$

where

$$
\begin{aligned}
f\left(u, v, \delta^{*}\right)= & \frac{\left[a_{13}\left(\lambda_{2}-a_{11}\right)-a_{12} a_{23}\right] u^{2}}{a_{12}\left(\lambda_{2}+1\right)}+\frac{\left[a_{14}\left(\lambda_{2}-a_{11}\right)-a_{12} a_{24}\right] u v}{a_{12}\left(\lambda_{2}+1\right)}-\frac{a_{12} a_{25} v^{2}}{a_{12}\left(\lambda_{2}+1\right)} \\
& +\frac{\left[b_{11}\left(\lambda_{2}-a_{11}\right)-a_{12} b_{21}\right] \delta^{*} u}{a_{12}\left(\lambda_{2}+1\right)}+\frac{\left[b_{12}\left(\lambda_{2}-a_{11}\right)-a_{12} b_{22}\right] \delta^{*} v}{a_{12}\left(\lambda_{2}+1\right)} \\
& +\frac{\left[b_{13}\left(\lambda_{2}-a_{11}\right)-a_{12} b_{23}\right] \delta^{*} u^{2}}{a_{12}\left(\lambda_{2}+1\right)}+\frac{\left[b_{14}\left(\lambda_{2}-a_{11}\right)-a_{12} b_{24}\right] \delta^{*} u v}{a_{12}\left(\lambda_{2}+1\right)}-\frac{a_{12} b_{25} \delta^{*} v^{2}}{a_{12}\left(\lambda_{2}+1\right)} \\
& +O\left(|u|,|v|,\left|\delta^{*}\right|\right)^{3}, \\
g\left(u, v, \delta^{*}\right)= & \frac{\left[a_{13}\left(1+a_{11}\right)+a_{12} a_{23}\right] u^{2}}{a_{12}\left(\lambda_{2}+1\right)}+\frac{\left[a_{14}\left(1+a_{11}\right)+a_{12} a_{24}\right] u v}{a_{12}\left(\lambda_{2}+1\right)}+\frac{a_{12} a_{25} v^{2}}{a_{12}\left(\lambda_{2}+1\right)}
\end{aligned}
$$




$$
\begin{aligned}
& +\frac{\left[b_{11}\left(1+a_{11}\right)+a_{12} b_{21}\right] \delta^{*} u}{a_{12}\left(\lambda_{2}+1\right)}+\frac{\left[b_{12}\left(1+a_{11}\right)+a_{12} b_{22}\right] \delta^{*} v}{a_{12}\left(\lambda_{2}+1\right)} \\
& +\frac{\left[b_{13}\left(1+a_{11}\right)+a_{12} b_{23}\right] \delta^{*} u^{2}}{a_{12}\left(\lambda_{2}+1\right)}+\frac{\left[b_{14}\left(1+a_{11}\right)+a_{12} b_{24}\right] \delta^{*} u v}{a_{12}\left(\lambda_{2}+1\right)}+\frac{a_{12} b_{25} \delta^{*} v^{2}}{a_{12}\left(\lambda_{2}+1\right)} \\
& +O\left(|u|,|v|,\left|\delta^{*}\right|\right)^{3}, \\
& u=a_{12}(\tilde{x}+\tilde{y}), \quad v=-\left(1+a_{11}\right) \tilde{x}+\left(\lambda_{2}-a_{11}\right) \tilde{y} .
\end{aligned}
$$

Applying the center manifold theorem to (10) at the origin in the limited neighborhood of $\delta^{*}=0$. The center manifold $W^{c}(0,0)$ can be approximately represented as

$$
W^{c}(0,0)=\left\{(\tilde{x}, \tilde{y}): \tilde{y}=a_{0} \delta^{*}+a_{1} \tilde{x}^{2}+a_{2} \tilde{x} \delta^{*}+a_{3} \delta^{* 2}+O\left(\left(|\tilde{x}|+\left|\delta^{*}\right|\right)^{3}\right)\right\},
$$

where $O\left(\left(|\tilde{x}|+\left|\delta^{*}\right|\right)^{3}\right)$ is a function with at least third orders in variables $\left(\tilde{x}, \delta^{*}\right)$.

By simple calculations for the center manifold, we have

$$
\begin{aligned}
& a_{0}=0, \\
& a_{1}=\frac{\left[a_{13}\left(1+a_{11}\right)+a_{12} a_{23}\right] a_{12}-\left[a_{14}\left(1+a_{11}\right)+a_{12} a_{24}\right]\left(1+a_{11}\right)+a_{25}\left(1+a_{11}\right)^{2}}{1-\lambda_{2}^{2}}, \\
& a_{2}=\frac{-\left[b_{11}\left(1+a_{11}\right)+a_{12} b_{21}\right] a_{12}+\left[b_{12}\left(1+a_{11}\right)+a_{12} b_{22}\right]\left(1+a_{11}\right)}{a_{12}\left(\lambda_{2}+1\right)^{2}}, \\
& a_{3}=0 .
\end{aligned}
$$

Now, consider the map restricted to the center manifold $W^{c}(0,0)$ as below:

$$
f: \tilde{x} \rightarrow-\tilde{x}+h_{1} \tilde{x}^{2}+h_{2} \tilde{x} \delta^{*}+h_{3} \tilde{x}^{2} \delta^{*}+h_{4} \tilde{x} \delta^{* 2}+h_{5} \tilde{x}^{3}+O\left(\left(|\tilde{x}|+\left|\delta^{*}\right|\right)^{4}\right),
$$

where

$$
\begin{aligned}
h_{1}= & \frac{\left[a_{13}\left(\lambda_{2}-a_{11}\right)-a_{12} a_{23}\right] a_{12}}{\left(\lambda_{2}+1\right)}-\frac{\left[a_{14}\left(\lambda_{2}-a_{11}\right)-a_{12} a_{24}\right]\left(1+a_{11}\right)}{\left(\lambda_{2}+1\right)}-\frac{a_{12} a_{25}\left(1+a_{11}\right)^{2}}{a_{12}\left(\lambda_{2}+1\right)}, \\
h_{2}= & \frac{\left[b_{11}\left(\lambda_{2}-a_{11}\right)-a_{12} b_{21}\right]}{\left(\lambda_{2}+1\right)}-\frac{\left[b_{12}\left(\lambda_{2}-a_{11}\right)-a_{12} b_{22}\right]\left(1+a_{11}\right)}{a_{12}\left(\lambda_{2}+1\right)}, \\
h_{3}= & \frac{\left[a_{13}\left(\lambda_{2}-a_{11}\right)-a_{12} a_{23}\right] 2 a_{2} a_{12}}{\left(\lambda_{2}+1\right)}+\frac{\left[a_{14}\left(\lambda_{2}-a_{11}\right)-a_{12} a_{24}\right]\left(\lambda_{2}-2 a_{11}-1\right) a_{2}}{\left(\lambda_{2}+1\right)} \\
& +\frac{2 a_{25}\left(1+a_{11}\right)\left(\lambda_{2}-a_{11}\right) a_{2}}{\left(\lambda_{2}+1\right)}+\frac{\left[b_{11}\left(\lambda_{2}-a_{11}\right)-a_{12} b_{21}\right] a_{1}}{\left(\lambda_{2}+1\right)} \\
& +\frac{\left[b_{12}\left(\lambda_{2}-a_{11}\right)-a_{12} b_{22}\right]\left(\lambda_{2}-a_{11}\right) a_{1}}{a_{12}\left(\lambda_{2}+1\right)}+\frac{\left[b_{13}\left(\lambda_{2}-a_{11}\right)-a_{12} b_{23}\right] a_{12}}{\left(\lambda_{2}+1\right)} \\
& -\frac{\left[b_{14}\left(\lambda_{2}-a_{11}\right)-a_{12} b_{24}\right]\left(1+a_{11}\right)}{\left(\lambda_{2}+1\right)}-\frac{b_{25}\left(1+a_{11}\right)^{2}}{\left(\lambda_{2}+1\right)}, \\
h_{4}= & \frac{\left[b_{11}\left(\lambda_{2}-a_{11}\right)-a_{12} b_{21}\right] a_{2}}{\left(\lambda_{2}+1\right)}+\frac{\left[b_{12}\left(\lambda_{2}-a_{11}\right)-a_{12} b_{22}\right]\left(\lambda_{2}-a_{11}\right) a_{2}}{a_{12}\left(\lambda_{2}+1\right)}, \\
h_{5}= & \frac{\left[a_{13}\left(\lambda_{2}-a_{11}\right)-a_{12} a_{23}\right] 2 a_{12} a_{1}}{\left(\lambda_{2}+1\right)}+\frac{\left[a_{14}\left(\lambda_{2}-a_{11}\right)-a_{12} a_{24}\right]\left(\lambda_{2}-2 a_{11}-1\right) a_{1}}{\left(\lambda_{2}+1\right)} \\
& +\frac{2 a_{25}\left(1+a_{11}\right)\left(\lambda_{2}-a_{11}\right) a_{1}}{\left(\lambda_{2}+1\right)} .
\end{aligned}
$$


According to the flip bifurcation, the discriminatory quantities $\gamma_{1}$ and $\gamma_{2}$ are given by

$$
\begin{aligned}
& \gamma_{1}=\left.\left(\frac{\partial^{2} f}{\partial \tilde{x} \partial \delta^{*}}+\frac{1}{2} \frac{\partial f}{\partial \delta^{*}} \frac{\partial^{2} f}{\partial \tilde{x}^{2}}\right)\right|_{(0,0)}, \\
& \gamma_{2}=\left.\left(\frac{1}{6} \frac{\partial^{3} f}{\partial \tilde{x}^{3}}+\left(\frac{1}{2} \frac{\partial^{2} f}{\partial \tilde{x}^{2}}\right)^{2}\right)\right|_{(0,0)} .
\end{aligned}
$$

After simple calculations, we obtain $\gamma_{1}=h_{2}$ and $\gamma_{2}=h_{5}+h_{1}^{2}$.

Analyzing the above and the flip bifurcation conditions discussed in [23], we write the following theorem.

Theorem 3.1 If $\gamma_{2} \neq 0$, and the parameter $\delta^{*}$ alters in the limiting region of the point $(0,0)$, then the system (7) passes through a flip bifurcation at the point $B\left(x^{*}, y^{*}\right)$. Also, the period2 points that bifurcate from the fixed point $B\left(x^{*}, y^{*}\right)$ are stable (resp., unstable) if $\gamma_{2}>0$ (resp., $\left.\gamma_{2}<0\right)$.

\subsection{Neimark-Sacker bifurcation}

Consider the system (2) with arbitrary parameter $\left(a, b, d, k, l, m, n, q, \delta_{2}\right) \in H_{B}$, which is described as follows:

$$
\left\{\begin{array}{l}
x \rightarrow x+\delta_{2}\left[a x\left(1-\frac{x}{k}\right)-\frac{b x y}{x+l}\right] \\
y \rightarrow y+\delta_{2}\left[1-\frac{m y}{n x+q}-d\right] y .
\end{array}\right.
$$

$B\left(x^{*}, y^{*}\right)$ is a fixed point of the system (11), where $x^{*}, y^{*}$ are given by (3) and

$$
\delta_{2}=-\frac{G}{H} .
$$

Consider the perturbation of (11)

$$
\left\{\begin{array}{l}
x \rightarrow x+\left(\delta_{2}+\delta\right)\left[a x\left(1-\frac{x}{k}\right)-\frac{b x y}{x+l}\right] \\
y \rightarrow y+\left(\delta_{2}+\delta\right)\left[1-\frac{m y}{n x+q}-d\right] y
\end{array}\right.
$$

where $|\delta| \ll 1$ is limited perturbation parameter.

The characteristic equation of map (12) at $B\left(x^{*}, y^{*}\right)$ is given by

$$
\lambda^{2}+p(\delta) \lambda+q(\delta)=0
$$

where

$$
\begin{aligned}
& p(\delta)=-2-G\left(\delta_{2}+\delta\right), \\
& q(\delta)=1+G\left(\delta_{2}+\delta\right)+H\left(\delta_{2}+\delta\right)^{2} .
\end{aligned}
$$

Since the parameters $\left(a, b, d, k, l, m, n, q, \delta_{2}\right) \in H_{B}$, the eigen values of $B\left(x^{*}, y^{*}\right)$ are a pair of complex conjugate numbers $\bar{\lambda}$ and $\lambda$ with modulus 1 by Proposition 2.4, where

$$
\bar{\lambda}, \lambda=\frac{-p(\delta) \mp i \sqrt{4 q(\delta)-p^{2}(\delta)}}{2} .
$$


Therefore

$$
\bar{\lambda}, \lambda=1+\frac{G\left(\delta_{2}+\delta\right)}{2} \mp \frac{i\left(\delta_{2}+\delta\right) \sqrt{4 H-G^{2}}}{2} .
$$

Now we have

$$
|\lambda|=(q(\delta))^{1 / 2}, \quad l=\left.\frac{d|\lambda|}{d \delta}\right|_{\delta=0}=-\frac{G}{2}>0
$$

When $\delta$ varies in a limited neighborhood of $\delta=0$, then $\bar{\lambda}, \lambda=\alpha \mp i \beta$, where

$$
\begin{aligned}
& \alpha=1+\frac{\delta_{2} G}{2}, \\
& \beta=\frac{\delta_{2} \sqrt{4 H-G^{2}}}{2} .
\end{aligned}
$$

The Neimark-Sacker bifurcation requires that when $\delta=0$, then $\bar{\lambda}^{j}, \lambda^{j} \neq 1(j=1,2,3,4)$, which is equivalent to $p(0) \neq-2,0,1,2$.

Since the parameter $\left(a, b, d, k, l, m, n, q, \delta_{2}\right) \in H_{B}$, therefore $p(0) \neq-2,2$. The only requirement is that $p(0) \neq 0,1$, from which it follows that

$$
G^{2} \neq 2 H, 3 H
$$

Let $u=x-x^{*}$ and $v=y-y^{*}$.

After the transformation of the fixed point $B\left(x^{*}, y^{*}\right)$ of system (12) to the point $(0,0)$, we have

$$
\left(\begin{array}{l}
u \\
v
\end{array}\right) \rightarrow\left(\begin{array}{c}
a_{11} u+a_{12} v+a_{13} u^{2}+a_{14} u v+O(|u|,|v|)^{3} \\
a_{21} u+a_{22} v+a_{23} u^{2}+a_{24} u v+a_{25} v^{2}+O(|u|,|v|)^{3}
\end{array}\right)
$$

where $a_{11}, a_{12}, a_{13}, a_{14}, a_{21}, a_{22}, a_{23}, a_{24}, a_{25}$ are given in (9) by substituting $\delta_{2}$ for $\delta_{2}+\delta$.

Now, we discuss the normal form of (14) when $\delta=0$.

Consider the translation

$$
\left(\begin{array}{l}
u \\
v
\end{array}\right)=T\left(\begin{array}{l}
\tilde{x} \\
\tilde{y}
\end{array}\right)
$$

where

$$
T=\left(\begin{array}{cc}
a_{12} & 0 \\
\alpha-a_{11} & -\beta
\end{array}\right)
$$

Taking $T^{-1}$ on both sides of (14), we get

$$
\left(\begin{array}{l}
\tilde{x} \\
\tilde{y}
\end{array}\right) \rightarrow\left(\begin{array}{cc}
\alpha & -\beta \\
\beta & \alpha
\end{array}\right)\left(\begin{array}{l}
\tilde{x} \\
\tilde{y}
\end{array}\right)+\left(\begin{array}{l}
\tilde{f}(\tilde{x}, \tilde{y}) \\
\tilde{g}(\tilde{x}, \tilde{y})
\end{array}\right)
$$


where

$$
\begin{aligned}
& \tilde{f}(\tilde{x}, \tilde{y})= \frac{a_{13}}{a_{12}} u^{2}+\frac{a_{14}}{a_{12}} u v+O(|u|,|v|)^{3}, \\
& \tilde{g}(\tilde{x}, \tilde{y})= \frac{\left[a_{13}\left(\alpha-a_{11}\right)-a_{12} a_{23}\right]}{a_{12} \beta} u^{2}+\frac{\left[a_{14}\left(\alpha-a_{11}\right)-a_{12} a_{24}\right]}{a_{12} \beta} u v \\
& \quad-\frac{a_{25}}{\beta} v^{2}+O(|u|,|v|)^{3}, \\
& u=a_{12} \tilde{x}, \quad v=\left(\alpha-a_{11}\right) \tilde{x}-\beta \tilde{y} .
\end{aligned}
$$

Now

$$
\begin{aligned}
& \tilde{f}_{\tilde{x} \tilde{x}}=2 a_{12} a_{13}+2 a_{14}\left(\alpha-a_{11}\right), \quad \tilde{f}_{\tilde{x} \tilde{y}}=-a_{14} \beta, \quad \tilde{f}_{\tilde{y} \tilde{y}}=0, \\
& \tilde{f}_{\tilde{x} \tilde{x} \tilde{x}}=0, \quad \tilde{f}_{\tilde{x} \tilde{y} \tilde{y}}=0, \quad \tilde{f}_{\tilde{x} \tilde{y} \tilde{y}}=0, \quad \tilde{f}_{\tilde{y} \tilde{y} \tilde{y}}=0, \\
& \tilde{g}_{\tilde{x} \tilde{x}}=\frac{2 a_{12}}{\beta}\left[a_{13}\left(\alpha-a_{11}\right)-a_{12} a_{23}\right]+\frac{2\left(\alpha-a_{11}\right)}{\beta}\left[a_{14}\left(\alpha-a_{11}\right)-a_{12} a_{24}\right] \\
& \\
&-\frac{2 a_{25}}{\beta}\left(\alpha-a_{11}\right)^{2}, \\
& \tilde{g}_{\tilde{x} \tilde{y}}=-\left[a_{14}\left(\alpha-a_{11}\right)-a_{12} a_{24}\right]+2 a_{25}\left(\alpha-a_{11}\right), \quad \tilde{g}_{\tilde{y} \tilde{y}}=-2 a_{25} \beta, \\
& \tilde{g}_{\tilde{x} \tilde{x} \tilde{x}}=0, \quad \tilde{g}_{\tilde{x} \tilde{x} \tilde{y}}=0, \quad \tilde{g}_{\tilde{x} \tilde{y} \tilde{y}}=0, \quad \tilde{g}_{\tilde{y} \tilde{y} \tilde{y}}=0 .
\end{aligned}
$$

According to the Neimark-Sacker bifurcation, the discriminatory quantity $s$ is given by

$$
s=-\operatorname{Re}\left[\frac{(1-2 \bar{\lambda}) \bar{\lambda}^{2}}{1-\lambda} \varphi_{11} \varphi_{20}\right]-\frac{1}{2}\left\|\varphi_{11}\right\|^{2}-\left\|\varphi_{02}\right\|^{2}+\operatorname{Re}\left(\bar{\lambda} \varphi_{21}\right)
$$

where

$$
\begin{aligned}
& \varphi_{20}=\frac{1}{8}\left[\left(\tilde{f}_{\tilde{x} \tilde{x}}-\tilde{f}_{\tilde{y} \tilde{y}}+2 \tilde{g}_{\tilde{x} \tilde{y}}\right)+i\left(\tilde{g}_{\tilde{x} \tilde{x}}-\tilde{g}_{\tilde{y} \tilde{y}}-2 \tilde{f}_{\tilde{x} \tilde{y}}\right)\right], \\
& \varphi_{11}=\frac{1}{4}\left[\left(\tilde{f}_{\tilde{x} \tilde{x}}+\tilde{f}_{\tilde{y} \tilde{y}}\right)+i\left(\tilde{g}_{\tilde{x} \tilde{x}}+\tilde{g}_{\tilde{y} \tilde{y}}\right)\right], \\
& \varphi_{02}=\frac{1}{8}\left[\left(\tilde{f}_{\tilde{x} \tilde{x}}-\tilde{f}_{\tilde{y} \tilde{y}}-2 \tilde{g}_{\tilde{x} \tilde{y}}\right)+i\left(\tilde{g}_{\tilde{x} \tilde{x}}-\tilde{g}_{\tilde{y} \tilde{y}}+2 \tilde{f}_{\tilde{x} \tilde{y}}\right)\right], \\
& \left.\varphi_{21}=\frac{1}{16}\left[\tilde{f}_{\tilde{x} \tilde{x} \tilde{x}}+\tilde{f}_{\tilde{x} \tilde{y} \tilde{y}}+\tilde{g}_{\tilde{x} \tilde{x} \tilde{y}}+\tilde{g}_{\tilde{y} \tilde{y} \tilde{y}}\right)+i\left(\tilde{g}_{\tilde{x} \tilde{x} \tilde{x}}+\tilde{g}_{\tilde{x} \tilde{y} \tilde{y}}-\tilde{f}_{\tilde{x} \tilde{x} \tilde{y}}-\tilde{f}_{\tilde{y} \tilde{y} \tilde{y}}\right)\right] .
\end{aligned}
$$

Analyzing the above and the Neimark-Sacker bifurcation conditions discussed in [23], we write the theorem as follows.

Theorem 3.2 If the condition (13) holds, $s \neq 0$ and the parameter $\delta$ alters in the limited region of the point $(0,0)$, then the system (12) passes through a Neimark-Sacker bifurcation at the point $B\left(x^{*}, y^{*}\right)$. Moreover, if $s<0$ (resp., $s>0$ ), then an attracting (resp., repelling) invariant closed curve bifurcates from the fixed point $B\left(x^{*}, y^{*}\right)$ for $\delta>0($ resp., $\delta<0)$.

\section{Numerical simulations}

To verify the theoretical analysis, we draw the bifurcation diagrams, the largest Lyapunov exponents, and the phase portraits for the system (2) with an initial value of $(x, y)=$ 


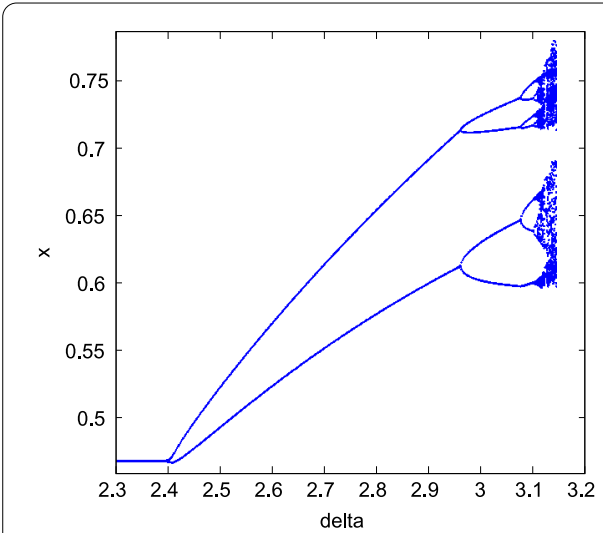

(a)

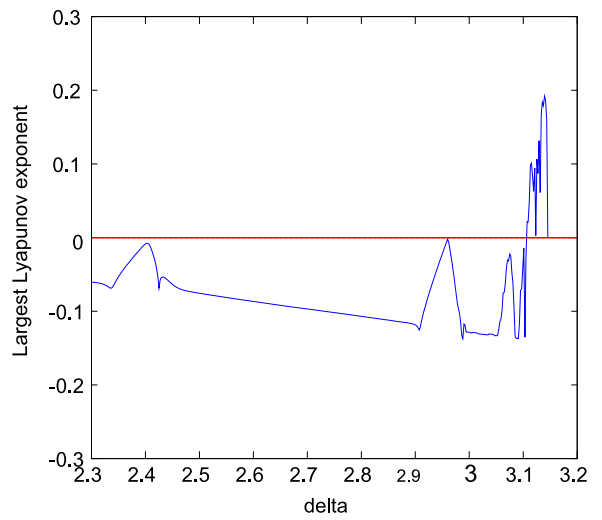

(b)

Figure 1 Bifurcation diagram and largest Lyapunov exponents of the system (2) for $a=0.2, b=0.12$, $d=0.14, k=4, I=3, m=0.1, n=0.2, q=0.5$, and $\delta$ covering [2.3, 3.2].

$(3.5,1.6)$. We discuss the following two cases by keeping the parameters $b=0.12, k=4$, $l=3, m=0.1, n=0.2, q=0.5$ as fixed and varying the parameters $a, d$ only.

Case 1: In this case, we take $a=0.2, d=0.14$ and obtain $\gamma_{1}=-3.10$ and $\gamma_{2}=8.59$. From Figure $1(\mathrm{a})$, it is observed that the fixed point $(0.46,5.10)$ of the system $(2)$ is stable for $\delta<2.406$, loses its stability, and a flip bifurcation appears at $\delta=2.406$. It shows that Theorem 3.1 is true.

The phase portraits show that there are orbits of period 2, 4,8 at $\delta=2.41,3.0,3.1$, respectively, and chaotic sets at $\delta=3.12,3.15$ (see Figure 2). Moreover, the largest Lyapunov exponents corresponding to $\delta=3.12$ and 3.15 are positive, confirming the existence of chaotic sets (see Figure 1(b)).

Case 2: In this case, we take $a=0.8, d=0.44$ and obtain $s=-0.00503$. From Figure 3(a), it is observed that the fixed point $(3.38,6.59)$ of the system (2) is stable for $\delta<3.066$, loses its stability, and a Neimark-Sacker bifurcation emerges at $\delta=3.066$. It indicates that Theorem 3.2 is satisfied.

The phase portraits in Figures 4 and 5 show that a smooth invariant circle bifurcates from the fixed point $(3.38,6.59)$ and its radius increases with the increase of $\delta$. There are windows of period 13, 26 at $\delta=3.34,3.36$, respectively, and chaotic attractors at $\delta=$ 3.38 and 3.4. Moreover, the largest Lyapunov exponents corresponding to $\delta=3.38$ and 3.4 are positive, confirming that we have chaotic sets (see Figure 3(b)).

\section{Conclusions}

In this paper, we investigated the stability and bifurcation analysis of discrete-time preypredator system with predator partially dependent on prey in the closed first quadrant $R_{+}^{2}$. The map undergoes a flip bifurcation and a Neimark-Sacker bifurcation at the fixed point under specific conditions when $\delta$ varies in a small neighborhood of $F_{B 1}$ or $F_{B 2}$ and $H_{B}$. Numerical simulations of the model display orbits of period 2, 4, 8 and chaotic sets in the case of a flip bifurcation; and a smooth invariant circle, period 13, 26 windows and chaotic attractors in the case of a Neimark-Sacker bifurcation. It signifies that the predator coexists with the prey at the period- $n$ orbits and a smooth invariant circle. 


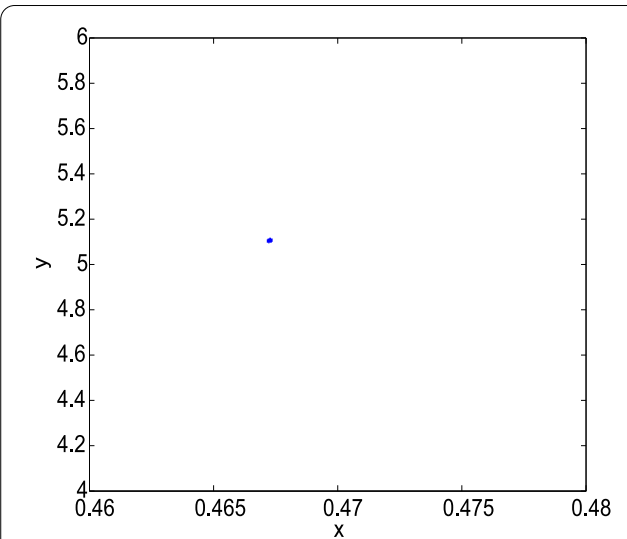

(a) $\delta=2.4$

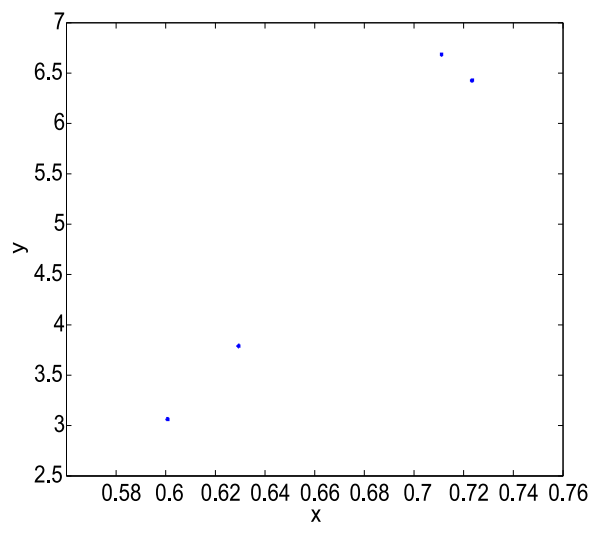

(c) $\delta=3.0$

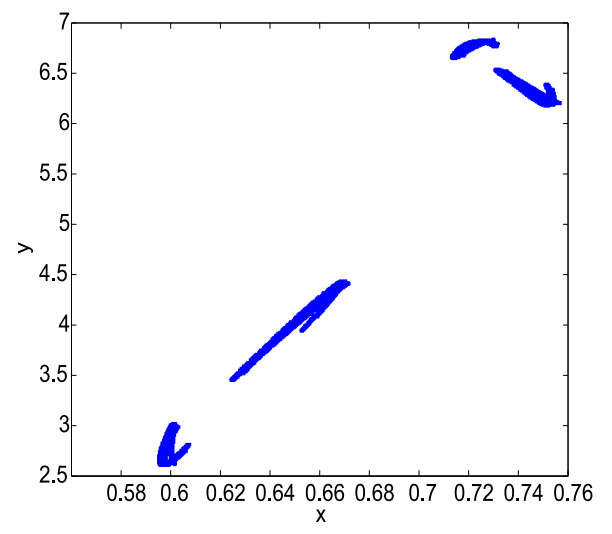

(e) $\delta=3.12$

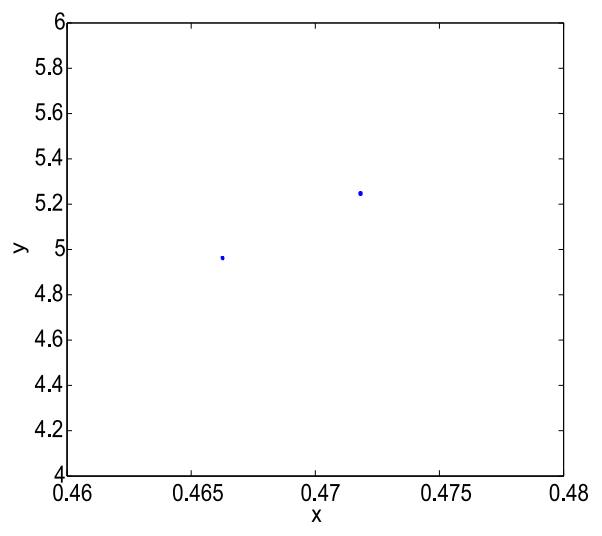

(b) $\delta=2.41$

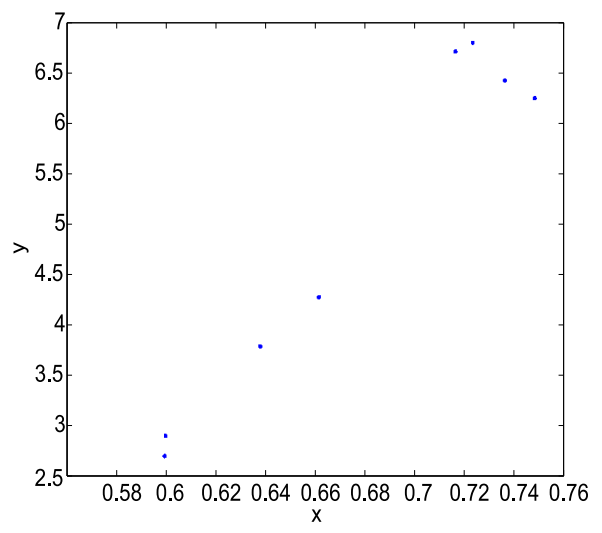

(d) $\delta=3.1$

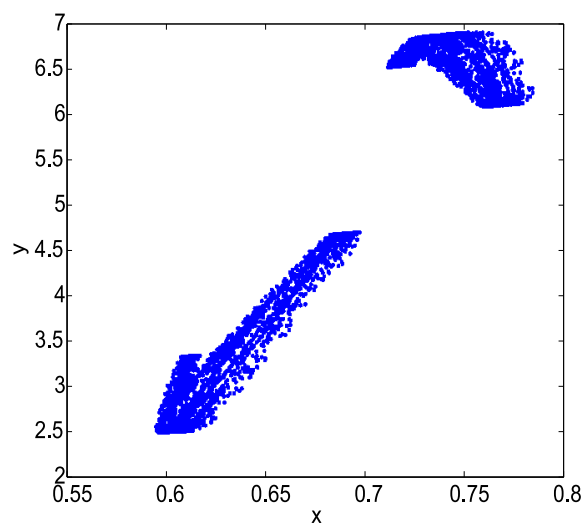

(f) $\delta=3.15$

Figure 2 Phase portraits for several values of $\delta$ from 2.4 to 3.15 related to Figure 1(a). 


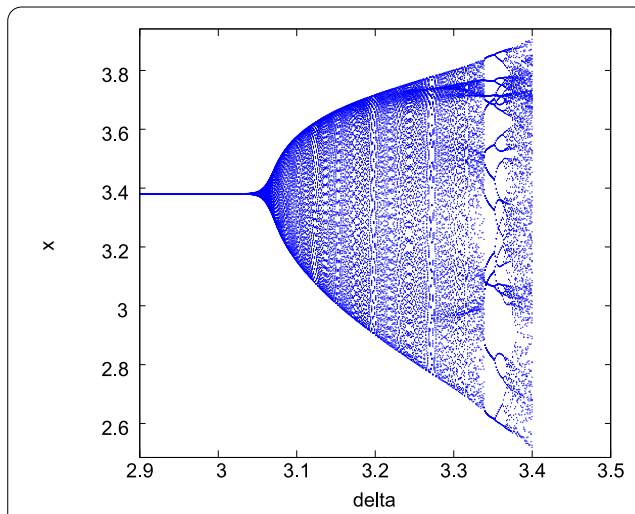

(a)

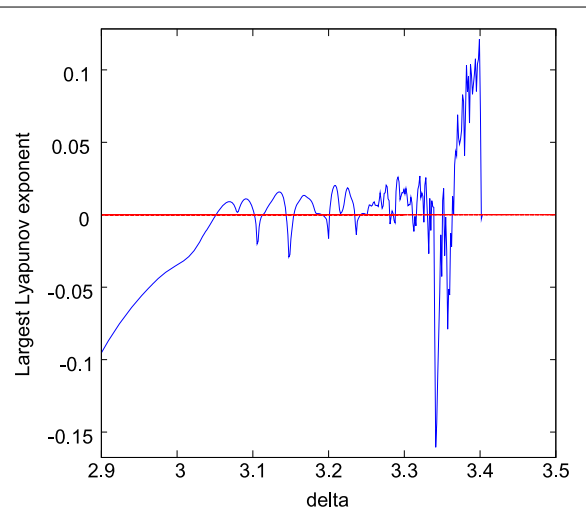

(b)

Figure 3 Bifurcation diagram and largest Lyapunov exponents of the system (2) for $a=0.8, b=0.12$, $d=0.44, k=4, l=3, m=0.1, n=0.2, q=0.5$, and $\delta$ covering $[2.9,3.5]$.

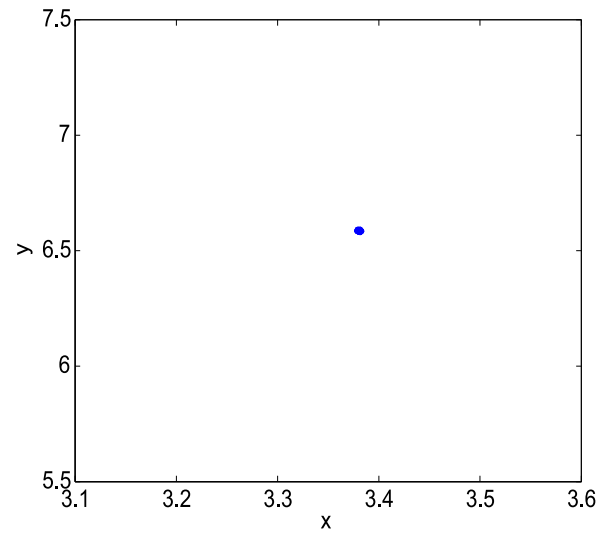

(a) $\delta=3.06$

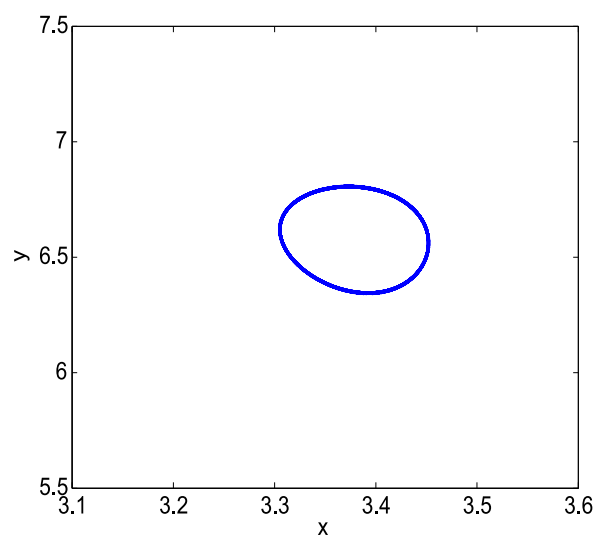

(c) $\delta=3.07$

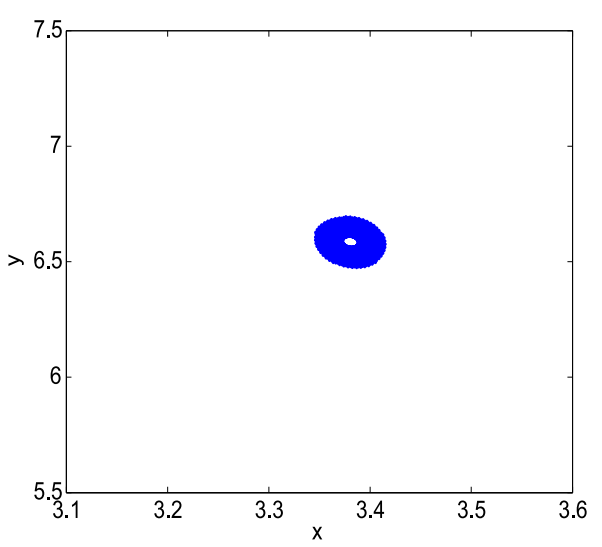

(b) $\delta=3.066$

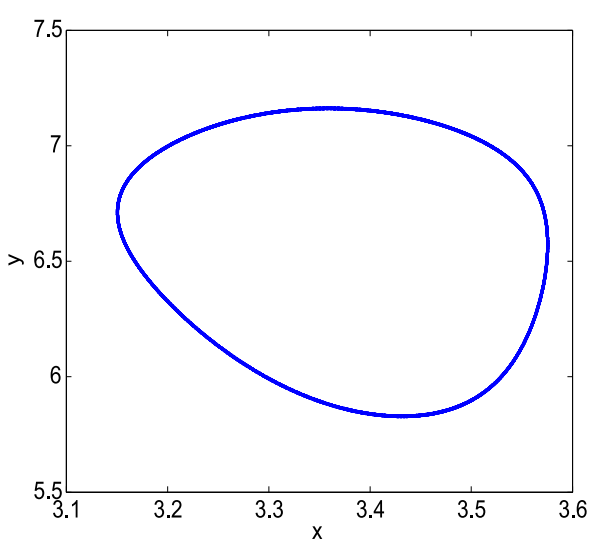

(d) $\delta=3.1$

Figure 4 Phase portraits for various values of $\delta$ from 3.06 to 3.1 related to Figure 3(a). 


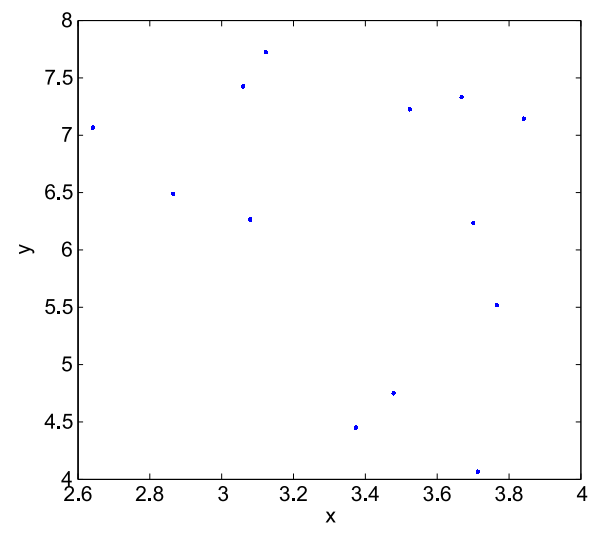

(a) $\delta=3.34$

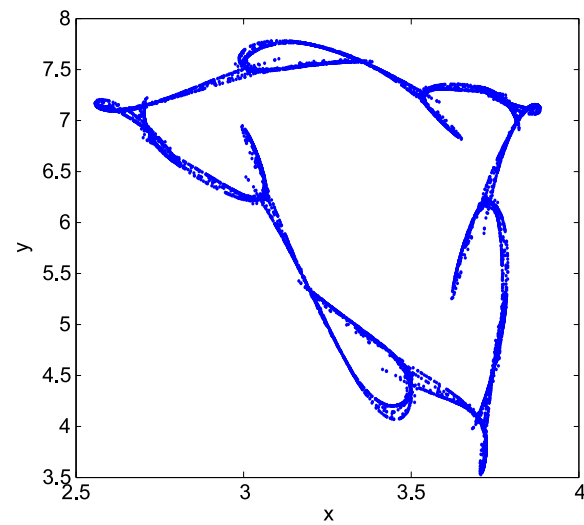

(c) $\delta=3.38$

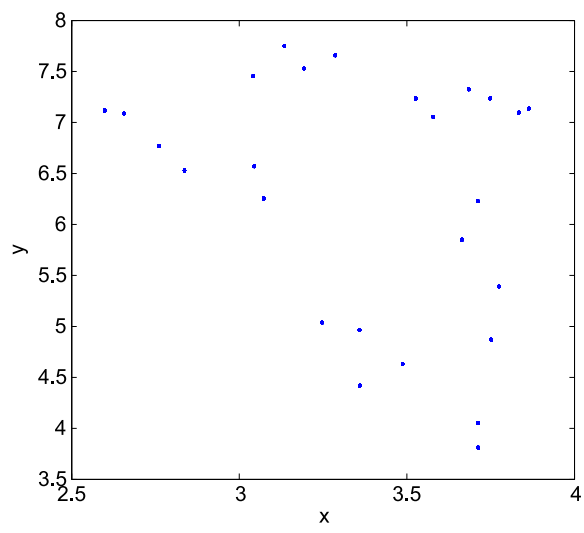

(b) $\delta=3.36$

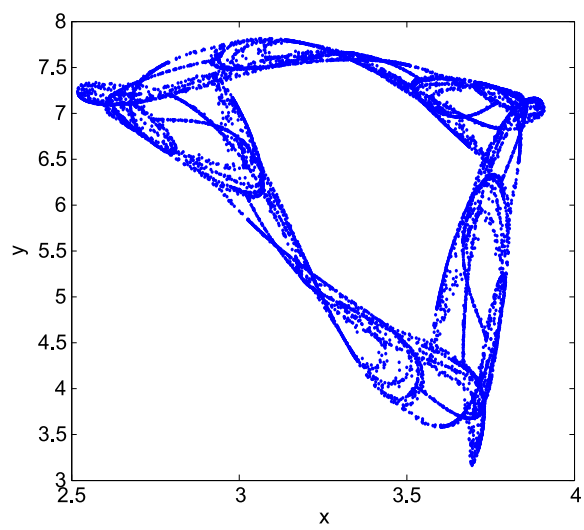

(d) $\delta=3.4$

Figure 5 Phase portraits for several values of $\delta$ from 3.34 to 3.4 related to Figure $3(\mathrm{a})$.

\section{Competing interests}

The authors declare that they have no competing interests.

\section{Authors' contributions}

All authors read and approved the manuscript

\section{Author details}

${ }^{1}$ Department of Applied Sciences, Khalsa College of Engineering and Technology, Amritsar, Punjab 143001, India. 2Department of Applied Sciences, ABV - Indian Institute of Information Technology and Management, Gwalior, MP 474015, India. ${ }^{3}$ Department of Applied Sciences, B.B.S.B. Engineering College, Fatehgarh Sahib, Punjab 24105, India.

\section{Acknowledgements}

The authors would like to thank the referee and reviewer for their constructive suggestions on improving the presentation of the paper.

Received: 2 April 2015 Accepted: 17 June 2015 Published online: 07 July 2015

\section{References}

1. Berryman, AA: The origins and evolution of predator-prey theory. Ecology 73(5), 1530-1535 (1992)

2. Lotka, AJ: Elements of Physical Biology (1925)

3. May, RM: Stability and Complexity in Model Ecosystems, vol. 6. Princeton University Press, Princeton (2001)

4. Volterra, V: Fluctuations in the abundance of a species considered mathematically. Nature 118, 558-560 (1926)

5. Agrawal, T, Saleem, M: Complex dynamics in a ratio-dependent two-predator one-prey model. Comput. Appl. Math. 34, 265-274 (2015)

6. Alebraheem, J, Hasan, YA: Dynamics of a two predator-one prey system. Comput. Appl. Math. 33, 767-780 (2014)

7. Dhar, J: A prey-predator model with diffusion and a supplementary resource for the prey in a two-patch environment. Math. Model. Anal. 9(1), 9-24 (2004)

8. Dhar, J, Jatav, KS: Mathematical analysis of a delayed stage-structured predator-prey model with impulsive diffusion between two predators territories. Ecol. Complex. 16, 59-67 (2013) 
9. Dubey, B: A prey-predator model with a reserved area. Nonlinear Anal., Model. Control 12(4), 479-494 (2007)

10. Freedman, H: Deterministic Mathematical Models in Population Ecology. HIFR Consulting Ltd, Edmonton (1980)

11. Jeschke, JM, Kopp, M, Tollrian, R: Predator functional responses: discriminating between handling and digesting prey. Ecol. Monogr. 72(1), 95-112 (2002)

12. Kooij, RE, Zegeling, A: A predator-prey model with Ivlev's functional response. J. Math. Anal. Appl. 198(2), 473-489 (1996)

13. Ma, W, Takeuchi, Y: Stability analysis on a predator-prey system with distributed delays. J. Comput. Appl. Math. 88(1), 79-94 (1998)

14. Sen, M, Banerjee, M, Morozov, A: Bifurcation analysis of a ratio-dependent prey-predator model with the Allee effect. Ecol. Complex. 11, 12-27 (2012)

15. Sinha, S, Misra, O, Dhar, J: Modelling a predator-prey system with infected prey in polluted environment. Appl. Math. Model. 34(7), 1861-1872 (2010)

16. Murray, JD: Mathematical Biology I: An Introduction. Interdisciplinary Applied Mathematics, vol. 17. Springer, New York (2002)

17. Robinson, C: Dynamical Systems: Stability, Symbolic Dynamics, and Chaos. CRC Press, Boca Raton (1998)

18. Agarwal, RP: Difference Equations and Inequalities: Theory, Methods, and Applications. CRC Press, Boca Raton (2000)

19. Agarwal, RP, Wong, PJ: Advanced Topics in Difference Equations. Springer, Berlin (1997)

20. Celik, C, Duman, O: Allee effect in a discrete-time predator-prey system. Chaos Solitons Fractals 40(4), 1956-1962 (2009)

21. Dhar, J, Singh, H, Bhatti, HS: Discrete-time dynamics of a system with crowding effect and predator partially dependent on prey. Appl. Math. Comput. 252, 324-335 (2015)

22. Gopalsamy, K: Stability and Oscillations in Delay Differential Equations of Population Dynamics. Springer, Berlin (1992)

23. Guckenheimer, J, Holmes, P: Nonlinear Oscillations, Dynamical Systems, and Bifurcations of Vector Fields. Applied Mathematical Sciences, vol. 42. Springer, New York (1983)

24. Liu, X, Xiao, D: Complex dynamic behaviors of a discrete-time predator-prey system. Chaos Solitons Fractals 32(1), 80-94 (2007)

25. Liu, X: A note on the existence of periodic solutions in discrete predator-prey models. Appl. Math. Model. 34(9), 2477-2483 (2010)

26. Huo, HF, Li, WT: Existence and global stability of periodic solutions of a discrete predator-prey system with delays. Appl. Math. Comput. 153(2), 337-351 (2004)

27. Chen, F: Permanence and global attractivity of a discrete multispecies Lotka-Volterra competition predator-prey systems. Appl. Math. Comput. 182(1), 3-12 (2006)

28. Liao, X, Zhou, S, Ouyang, Z: On a stoichiometric two predators on one prey discrete model. Appl. Math. Lett. 20(3) 272-278 (2007)

29. Fan, YH, Li, WT: Permanence for a delayed discrete ratio-dependent predator-prey system with Holling type functional response. J. Math. Anal. Appl. 299(2), 357-374 (2004)

30. Chen, Y, Changming, S: Stability and Hopf bifurcation analysis in a prey-predator system with stage-structure for prey and time delay. Chaos Solitons Fractals 38(4), 1104-1114 (2008)

31. Gakkhar, S, Singh, A: Complex dynamics in a prey predator system with multiple delays. Commun. Nonlinear Sci. Numer. Simul. 17(2), 914-929 (2012)

32. He, Z, Lai, X: Bifurcation and chaotic behavior of a discrete-time predator-prey system. Nonlinear Anal., Real World Appl. 12(1), 403-417 (2011)

33. Hu, Z, Teng, Z, Zhang, L: Stability and bifurcation analysis of a discrete predator-prey model with nonmonotonic functional response. Nonlinear Anal., Real World Appl. 12(4), 2356-2377 (2011)

34. Jing, Z, Yang, J: Bifurcation and chaos in discrete-time predator-prey system. Chaos Solitons Fractals 27(1), 259-277 (2006)

35. Zhang, $\mathrm{CH}$, Yan, XP, Cui, GH: Hopf bifurcations in a predator-prey system with a discrete delay and a distributed delay. Nonlinear Anal., Real World Appl. 11(5), 4141-4153 (2010)

36. Leslie, P: Some further notes on the use of matrices in population mathematics. Biometrika 35, $213-245$ (1948)

37. Leslie, P: A stochastic model for studying the properties of certain biological systems by numerical methods. Biometrika 45, 16-31 (1958)

38. Ricklefs, RE, Miller, GL: Ecology. Freeman, New York (2000)

\section{Submit your manuscript to a SpringerOpen ${ }^{\circ}$ journal and benefit from:}

- Convenient online submission

Rigorous peer review

- Immediate publication on acceptance

- Open access: articles freely available online

- High visibility within the field

- Retaining the copyright to your article 